\title{
ORIGINAL ARTICLE Pyrethroid insecticides and their environmental degradates in repeated duplicate-diet solid food samples of 50 adults
}

\author{
Marsha K. Morgan ${ }^{1}$, Denise K. MacMillan ${ }^{2}$, Dan Zehr $^{2}$ and Jon R. Sobus ${ }^{1}$
}

Previous research has reported concurrent levels of pyrethroid insecticides and their environmental degradates in foods. These data raise concerns about using these same pyrethroid degradates found in the diet as urinary biomarkers of exposures in humans. The primary objective was to quantify levels of selected pyrethroids and their environmental degradates in duplicate-diet solid food samples of 50 adults over a six-week monitoring period. The study was conducted at the US EPA's Human Studies Facility in North Carolina and at participants' residences in 2009-2011. Participants collected duplicate-diet solid food samples on days 1 and 2 during weeks 1, 2, and 6 of the monitoring period. These samples were collected over three consecutive time periods each sampling day. A total of 782 food samples were homogenized and analyzed by LC/MS/MS for seven pyrethroids (bifenthrin, $\lambda$-cyhalothrin, cyfluthrin, cypermethrin, cis-deltamethrin, esfenvalerate, and cis/trans-permethrin) and six pyrethroid degradates. Results showed that $49 \%$ and $2 \%$ of all the samples contained at least one target pyrethroid or pyrethroid degradate, respectively. Cis/trans-permethrin (20\%) and bifenthrin (20\%) were the most frequently detected pyrethroids. The results suggest that the pyrethroid degradates were likely not present in sufficient levels in the diet to substantially impact the adults' urinary biomarker concentrations.

Journal of Exposure Science and Environmental Epidemiology (2018) 28, 40-45; doi:10.1038/jes.2016.69; published online 14 December 2016

Keywords: adults; degradates; dietary; food; pyrethroids; temporal

\section{INTRODUCTION}

Pyrethroids are a class of insecticides that are used for crop protection worldwide. ${ }^{1,2}$ These lipophilic insecticides account for more than $17 \%$ of the global agrochemical market. ${ }^{1}$ In the United States (US), at least 18 different pyrethroid insecticides are currently registered by the US Environmental Protection Agency (EPA) for use on domestic crops for human consumption. ${ }^{3}$ However, no published data are currently available on the total amount of pyrethroid insecticides that are applied on US croplands, including residential gardens, each year. ${ }^{3}$

Research has shown that humans can be exposed to pyrethroids through the inhalation, ingestion, and dermal routes. ${ }^{2,4,5}$ However, dietary ingestion is likely the major exposure route to these insecticides in the general US adult population. ${ }^{4-5}$ This is supported by several studies reporting measureable residues of a number of different current-use pyrethroids in duplicate-diet samples or in individual food items (e.g., lettuce, blueberries, butter, and sour cream)..$^{6-14}$ In these studies, pyrethroid insecticides were generally detected at much higher levels in solid foods compared to liquid foods (e.g. beverages). $6,10,13,14$ Current-use pyrethroids detected in these solid food samples included bifenthrin, cyfluthrin, $\lambda$-cyhalothrin, cypermethrin, cis-deltamethrin, esfenvalerate, and cis/transpermethrin.

Few published data are available on the concurrent levels of these pyrethroid insecticides and their environmental degradates in foods. ${ }^{7,11}$ In a recent study by Chen et al., ${ }^{11}$ they reported measureable levels of cypermethrin, $\lambda$-cyhalothrin, cis-deltamethrin, esfenvalerate, and/or permethrin, and their common degradation product, 3-phenoxybenzoic acid (3-PBA), in eight different produce samples, collected in California between 2010 and 2011. This information raises concerns about using 3-PBA and perhaps other pyrethroid degradates found in the diet as urinary biomarkers of exposure in humans.

After oral ingestion, pyrethroids are metabolized quickly and are mainly renally eliminated as polar metabolites (e.g., 3-PBA) in humans with an urinary excretion half-life of less than 10 h. $^{15-17}$ For the pyrethroid degradates, no published (oral) toxicokinetic data exist based on human exposure studies. Previous biomonitoring studies have found several different pyrethroid metabolites (i.e., 3-PBA and cis/trans-3-(2,2-dichlorovinyl)-2,2-dimethylcyclopropane carboxylic acid (cis/trans-DCCA)) in the urine of the general US adult population.,18-21 it is currently unknown whether pyrethroid degradates in the diet are substantially contributing to pyrethroid metabolite levels in the urine of adults in the general US population.

The Pilot Study to Estimate Human Exposures to Pyrethroids using an Exposure Reconstruction Approach (Ex-R study) investigated the temporal exposures (over 6 weeks) of 50 North Carolina (NC) adults to selected pyrethroid insecticides and their environmental degradates in several different media (e.g., food, drinking water, dust, and floor wipes) at homes in 2009-2011.21 In this current work, we quantified the levels of seven current-use pyrethroids and six of their environmental degradates (Table 1) in 782 duplicate-diet solid food samples of 50 Ex-R adults over a six-

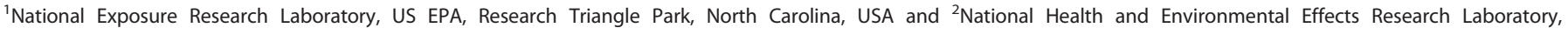

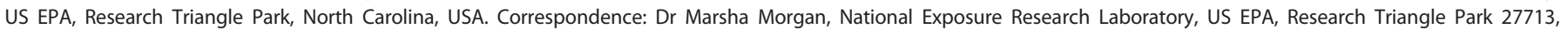
North Carolina, USA.

Tel.: +1 919541 2598. Fax: +1 9195410905.

E-mail: morgan.marsha@epa.gov

Received 10 May 2016; accepted 25 October 2016; published online 14 December 2016 
Table 1. Target pyrethroid insecticides and their environmental degradates measured in the duplicate-diet solid food samples.

\begin{tabular}{l}
\hline Pyrethroid \\
\cline { 2 - 4 } \\
\cline { 2 - 4 }
\end{tabular}

week monitoring period. We also estimated the maximum daily dietary intake doses of the adults to each target pyrethroid.

\section{MATERIALS AND METHODS}

\section{Study Cohort}

A detailed description of the Ex-R study design and sampling methodology can be found in Morgan et al. ${ }^{21}$ Briefly, this study was performed at the US EPA's Human Subjects Facility (HSF) located in Chapel Hill, NC and at the participants' residences within a 40 mile radius of the HSF. ${ }^{21}$ Recruitment of adult participants began in October 2009 and ended in March 2011. Field sampling occurred at the participants' residences from November 2009 to May 2011. The adults, ages $19-50$ years old, collected their own duplicate-diet solid food samples and completed daily food diaries over a 6-week monitoring period. All protocols and procedures for the Ex-R study were approved by the University of North Carolina's Institutional Review Board (study number: 09-0741). ${ }^{21}$ In addition, the adults signed informed consent forms before participating.

\section{Collection of Duplicate-Diet Solid Food Samples}

The duplicate-diet solid food samples consisted of duplicate amounts of all the consumed solid foods (excluding beverages) that the participants' ate on day 1 (Sunday) and day 2 (Monday) during weeks 1, 2, and 6 of the sixweek monitoring period. ${ }^{21}$ These samples were collected by each participant over three, consecutive time periods (period 1=4:00 am 11:00 am; period 2 = 11:00 am - 5:00 pm; and period 3= 5:00 pm - 4:00 am) each sampling day. Up to 18 individual food samples (6 per sampling week) were collected by each participant over the monitoring period. Solid foods were defined as any edible items that were solid, semi-solid, or contained solids (e.g., hamburger, salad, vegetable soup, smoothie, and ice cream). Inedible parts of foods such as chicken bones, banana peels, and apple cores as well as medicines/vitamins were excluded from these samples. The participants also completed daily food diaries that listed the specific food items that they consumed during each sampling time period.

For each food sample, the participants placed duplicate amounts of their consumed solid food items into a re-sealable polyethylene bag $(31 \times 31 \mathrm{~cm}$; Uline Shipping Supply Specialists, Pleasant Prairie, WI $)$ and then into a larger re-sealable polyethylene bag $(41 \times 41 \mathrm{~cm})$. The collection date of each food sample was written with a pen on the provided label on the outside polyethylene bag. The food samples were stored in portable thermoelectric coolers (Princess International, Brooklyn, NY, USA or Vinotemp, Irvine, CA, USA) until the participants returned the coolers to the HSF on day 3 (Tuesday) between 8:00 am-11:00 am of each sampling week. ${ }^{21}$ At the HSF, US EPA researchers weighed each food sample. These researchers then transported the food samples in study coolers with blue ice to an US EPA laboratory in Research Triangle Park (RTP), NC, USA.

\section{Food Sample Preparation, Extraction, and Analysis}

Food samples were homogenized (for up to $1 \mathrm{~min}$ ) using a vertical cutter mixer (Robot Coupe R4N-D or R10-Ultra) or a high-speed blender (Waring MBB518 Professional Food/Beverage). The blender was only used for a few types of food items (i.e., soups and purees). Samples containing a single food item with a smooth texture (i.e., peanut butter and ice cream) were not homogenized. Up to six replicates ( $12 \mathrm{~g}$ each) of each food sample were then transferred into separate, pre-cleaned amber glass jars $(30 \mathrm{ml})$ with lids. All replicates were stored in US EPA laboratory freezers $\left(\leqslant-20^{\circ} \mathrm{C}\right)$ until analysis.

The homogenized food samples were extracted using the QuEChERS (quick, easy, cheap, effective, rugged, and safe) technique that was modified for use in complex food mixtures. ${ }^{2,23}$ For each study sample, a $12 \mathrm{~g}$ replicate was thawed, and a $2 \mathrm{~g}$ aliquot was removed and transferred to a vial. The $2 \mathrm{~g}$ aliquot was then spiked with internal standards $\left({ }^{13} \mathrm{C}_{6}\right.$ cypermethrin, ${ }^{13} \mathrm{C}_{6}-\lambda$-cyhalothrin, ${ }^{13} \mathrm{C}_{6}$-cis-permethrin, ${ }^{13} \mathrm{C}_{6}$-transpermethrin, ${ }^{13} \mathrm{C}_{6}-3-\mathrm{PBA},{ }^{13} \mathrm{C}_{6}$-4-fluoro-3-phenoxybenzoic acid $\left({ }^{13} \mathrm{C}_{6}-4-\mathrm{F}-3-\right.$ PBA), and ${ }^{13} \mathrm{C}_{6}-\mathrm{d}_{1}$-cis-DCCA [Cambridge Isotope Laboratories, Andover, MA, USA]) and $2 \mathrm{ml}$ of acetonitrile was added. The sample was mechanically shaken for 1-2 min. to completely wet the contents, and then $\mathrm{MgSO}_{4}$ $(800 \mathrm{mg})$ and $\mathrm{NaCl}(200 \mathrm{mg})$ were added. The sample was vortexed to fully disperse the solids in the mixture, and then centrifuged at $4000 \mathrm{rpm}$ $(3220 \times \mathrm{g})$ for $5 \mathrm{~min}$. Next, an aliquot of the supernatant $(1 \mathrm{ml})$ was added to a clean-up tube containing $150 \mathrm{mg} \mathrm{MgSO}_{4} /$ carbon black (Supelclean ENVI-carb 120/400 (Supelco, Sigma-Aldrich, Bellefonte, PA, USA, 30:1; w/w) and $300 \mathrm{mg}$ bulk C-18 (Discovery DSC-C18, Supelco, Sigma-Aldrich). The clean-up tube was vortexed for $30 \mathrm{~s}$. and then centrifuged at 15,000 rpm $(26,000 \mathrm{~g})$ for $10 \mathrm{~min}$. An aliquot $(300 \mu \mathrm{l})$ of the resulting extract was diluted 1:1 with $0.1 \%$ formic acid, filtered through a PTFE filter $(0.2 \mu \mathrm{m})$, and stored at $\sim 4{ }^{\circ} \mathrm{C}$ until analysis.

The extracts were analyzed to quantify levels of the target pyrethroids and their environmental degradates (Table 1) using electrospray ionization liquid chromatography/tandem mass spectrometry (LC/MS/MS) on a 4000 QTrap linear ion trap mass spectrometer (AB Sciex, Framingham, MA, USA) in positive (pyrethroids) or negative (degradates) ion modes. All analytical standards were purchased from Chem Services (West Chester, PA, USA) with the exception of 4-F-3-PBA (Cambridge Isotope Laboratories, Andover, MA, USA) and 2-methyl-3-phenylbenzoic acid (MPA) (FMC Agricultural Products Group, Philadelphia, PA, USA). Instrument parameters are given in Supplementary Table 1. Separation was performed using a Hypersil Gold $50 \times 2.1 \mathrm{~mm}$ column $(1.9 \mu)$ (Thermo Fisher Scientific, Waltham, MA, USA) with gradient elution (Supplementary Table 2). The mobile phase used for the parent pyrethroids consisted of (A) $25: 755 \mathrm{~mm}$ ammonium acetate:methanol with $0.1 \%$ formic acid, and (B) $5 \mathrm{~mm}$ ammonium acetate in methanol with $0.1 \%$ formic acid. For the pyrethroid degradates, the mobile phases were the same as mentioned above, except formic acid was not included. Multiple reaction monitoring with two characteristic transitions per target analyte was used for definitive identification (Supplementary Table 3). Quantitation was obtained by internal calibration using isotope dilution and matrix-matched curves made by spiking the control matrix with internal standards and target analytes prior to extraction. The limits of quantitation (LOQs) for the target analytes were set at the concentration of the lowest calibration standard. Estimated limits of detection (LODs) were set at concentrations that gave a signal-to-noise ratio of at least 3 and were $\sim 2-5$ times below LOQs. The LODs and LOQs for the target analytes are provided in Table 2. Calibration ranges were from the LOQ to $200 \mathrm{ng} / \mathrm{g}$ for all analytes. Curves were constructed using quadratic equations with $1 / x$ weighting. Additional details of the method validation are included in the Supplementary Information section.

\section{Quality Assurance and Quality Control Procedures}

Field and laboratory quality control samples were used to determine the overall quality of food sample collection, processing, extraction, and analysis. A control food mixture was prepared in-house to be used as the medium for the field/laboratory blank and spike samples. Approximately $5 \mathrm{~kg}$ of the control food mixture (5\% fat) was made using a slightly modified method by Rosenblum et al. ${ }^{24}$ The food mixture contained applesauce, bread, cake, cereal, cheese, fruit cocktail, lunchmeat, 
Table 2. Estimated limits of detection (LODs) and limits of quantitation (LOQs) for the target analytes in the duplicate-diet solid food samples.

\begin{tabular}{|c|c|c|}
\hline Target analyte & $L O D(n g / g)$ & $L O Q(n g / g)$ \\
\hline \multicolumn{3}{|l|}{ Pyrethroid insecticide } \\
\hline Bifenthrin & 0.05 & 0.10 \\
\hline Cyfluthrin & 1.0 & 2.5 \\
\hline$\lambda$-Cyhalothrin & 0.30 & 1.0 \\
\hline Cypermethrin & 0.30 & 1.0 \\
\hline cis-Deltamethrin & 0.05 & 0.25 \\
\hline Esfenvalerate & 0.30 & 1.0 \\
\hline cis-Permethrin & 0.10 & 0.25 \\
\hline trans-Permethrin & 0.10 & 0.25 \\
\hline \multicolumn{3}{|l|}{ Pyrethroid degradate } \\
\hline 4-F-3-PBA & 0.10 & 0.25 \\
\hline 3-PBA & 0.30 & 2.5 \\
\hline cis-DBCA & 0.30 & 1.0 \\
\hline cis-DCCA & 0.30 & 1.0 \\
\hline trans-DCCA & 0.30 & 1.0 \\
\hline MPA & 0.30 & 1.0 \\
\hline \multicolumn{3}{|c|}{$\begin{array}{l}\text { Abbreviations: 3-PBA (3-phenoxybenzoic acid); 4-F-3-PBA (4-fluoro-3- } \\
\text { phenoxybenzoic acid); cis-DBCA (cis-3-(2,2-dibromovinyl)-2,2-dimethylcy- } \\
\text { clopropane carboxylic acid); cis-DCCA (cis-3-(2,2-dichlorovinyl)-2,2- } \\
\text { dimethylcyclopropane carboxylic acid); trans-DCCA (trans-3-(2,2-dichloro- } \\
\text { vinyl)-2,2-dimethylcyclo-propane carboxylic acid; MPA (2-methyl-3- } \\
\text { phenylbenzoic acid). }\end{array}$} \\
\hline
\end{tabular}

margarine, mayonnaise, pizza, potato chips, and vegetables that were purchased from local grocery stores. Organic food items were used (when available) to reduce background contamination of pyrethroid insecticides. The control food mixture was homogenized in $1 \mathrm{~kg}$ batches using a vertical cutter mixer (Robot Coupe R10-Ultra). Replicates of control food $(12 \mathrm{~g}$ each) were placed into pre-cleaned amber glass jars $(30 \mathrm{ml})$ and stored in $\leqslant-20^{\circ} \mathrm{C}$ in freezers.

The field QC samples consisted of blanks and three spikes (low, medium, and high levels) that were assigned to $10 \%$ of the study participants during days 1-2 of each sampling week. A blank or spike sample contained $12 \mathrm{~g}$ of the control food mixture (above) that was spiked with internal standards by using $100 \mu \mathrm{l}$ of a mixed standard $(500 \mathrm{ng} / \mathrm{ml}$ in methanol) for a final concentration of $4.2 \mathrm{ng} / \mathrm{g}$. For the field spike samples only, they were also spiked with 20,100 , or $200 \mu \mathrm{l}$ of a solution (in methanol) of the target pyrethroids for a final concentration of $0.9,4.5$, or $8.9 \mathrm{ng} / \mathrm{g}$ and the pyrethroid degradates for a final concentration of $4.5,22.5$, or $45 \mathrm{ng} / \mathrm{g}$, respectively. The concentration of the mixed standard used for spiking the field samples was $500 \mathrm{ng} / \mathrm{ml}$ for the pyrethroids and $2500 \mathrm{ng} / \mathrm{ml}$ for the degradates. Each field blank or field spike was stirred thoroughly with a spatula prior to being transferred into a re-sealable polyethylene bag $(8 \times 13 \mathrm{~cm})$ and then into another polyethylene bag $(8 \times 13 \mathrm{~cm})$. These field QC samples were placed into a portable thermoelectric cooler (plugged into an electric wall outlet) in a study room at the HSF during each sampling period (Sunday-Monday). The samples were stored in EPA laboratory freezers $\left(\leqslant-20^{\circ} \mathrm{C}\right)$ until analysis. The field blanks $(n=18)$ were below the LODs for all pyrethroids and their environmental degradates in food, except for cis-permethrin and trans-permethrin that co-occurred in two samples. For the medium and high field spike samples, mean percent recoveries of the target analytes were within empirically determined percent recovery acceptance limits, except for $\lambda$-cyhalothrin, cis-permethrin, trans-permethrin, 3-PBA, and cis-DBCA (Supplementary Table 4). For the low field spike samples, the recovery results were inconsistent and highly variable for all analytes (data not shown) most likely due to the low spiked volume $(20 \mu \mathrm{l})$ which caused incomplete mixing of the samples. A detailed discussion of the field spike results can be found in the Supplementary Information section.

The laboratory (batch) QC samples consisted of laboratory blanks, sample duplicates (duplicate extraction of a study sample), analytical duplicates (duplicate analysis of a study sample extract), laboratory control samples (LCS; spiked control mixture) and matrix spikes (MSS; spiked study samples). The LCS and MSS were spiked at $10 \mathrm{ng} / \mathrm{g}$. The laboratory blanks $(n=50)$ were all below the LODs for the target analytes in food, except for
Table 3. Levels of pyrethroid insecticides and their environmental degradates $(\mathrm{ng} / \mathrm{g})$ in the duplicate-diet solid food samples.

\begin{tabular}{lccccc}
\hline Target analyte & $\mathrm{N}^{\mathrm{a}, \mathrm{b}}$ & $\%^{\mathrm{c}}$ & 90th $^{\mathrm{d}}$ & $95 \mathrm{th}^{\mathrm{d}}$ & Maximum \\
\hline $\begin{array}{l}\text { Pyrethroid insecticide } \\
\text { Bifenthrin }\end{array}$ & 774 & 20 & 0.63 & 1.8 & 13.9 \\
Cyfluthrin & 781 & 1 & - & - & 103 \\
$\lambda$-Cyhalothrin & 781 & 2 & - & - & 27.7 \\
Cypermethrin & 781 & 7 & - & 1.6 & 154 \\
cis-Deltamethrin & 778 & 17 & 0.82 & 1.9 & 16.3 \\
Esfenvalerate & 780 & 2 & - & - & 358 \\
cis-Permethrin & 780 & 19 & 0.84 & 5.2 & 111 \\
trans-Permethrin & 774 & 21 & 0.90 & 5.4 & 172 \\
& & & & & \\
Pyrethroid degradate & & & & & \\
4-F-3-PBA & 781 & $<1$ & - & - & 0.4 \\
3-PBA & 780 & $<1$ & - & - & 2.3 \\
cis-DBCA & 779 & $<1$ & - & - & 1.0 \\
cis-DCCA & 782 & 1 & - & - & 8.3 \\
trans-DCCA & 782 & 2 & - & - & 38.3 \\
MPA & 780 & 0 & - & - & - \\
\hline AbA &
\end{tabular}

Abbreviations: 3-PBA (3-phenoxybenzoic acid); 4-F-3-PBA (4-fluoro-3phenoxybenzoic acid); cis-DBCA (cis-3-(2,2-dibromovinyl)-2,2-dimethylcyclopropane carboxylic acid); cis-DCCA (cis-3-(2,2-dichlorovinyl)-2,2dimethylcyclopropane carboxylic acid); trans-DCCA (trans-3-(2,2-dichlorovinyl)-2,2-dimethylcyclo-propane carboxylic acid; MPA (2-methyl-3phenylbenzoic acid). ${ }^{a}$ Number of samples $(n=782)$. ${ }^{b}$ Missing data due to laboratory error. ${ }^{\mathrm{C}}$ Percentage of analytes at or above the limit of detection by analyte. ${ }^{\mathrm{d}}$ Percentile.

cis-permethrin in one sample and trans-permethrin in three samples. Mean values for the laboratory blanks were below the LOD for each isomer, so no background correction was made. Mean relative percent differences between sample duplicates or analytical duplicates for the parent pyrethroids were less than $7 \%$ in the food samples. For the pyrethroid degradates, mean relative percent differences between the sample duplicates or analytical duplicates were less than $1 \%$. Mean percent recoveries for the LCS and MSS were within the empirically determined percent recovery acceptance limits for all target analytes (Supplementary Table 4). In addition, the mean percent recoveries of the target analytes for the MSS were within 5\% of the mean percent recoveries for the LCS, except for bifenthrin. For this analyte, the mean percent recovery for the MSS was $15 \%$ lower than the mean percent recovery for the LCS.

\section{Statistical Analyses}

For each target analyte, all data values below the LOD in the food samples were replaced by the LOD $/ \sqrt{2}$ as described in Verbovsek. ${ }^{25}$ Descriptive statistics were calculated for the analytes in the food samples using JMP version 12.1 (SAS Institute, Cary, NC, USA).

The estimated maximum daily dietary intake doses ( $\mathrm{ng} / \mathrm{kg} / \mathrm{day})$ of the Ex-R adults to each target pyrethroid insecticide $(D)$ was calculated using the following equation:

$$
D=\frac{\sum_{t=1}^{3}\left(F_{t} \times M_{t}\right)}{B}
$$

In this equation, $\mathrm{Ft}$ (concentration $(\mathrm{ng} / \mathrm{g})$ of each target pyrethroid in a food sample) was multiplied by $M_{t}$ (mass of the same food sample $(\mathrm{g})$ ). The subscript $\mathrm{t}$ denotes whether the sample was the first, second, or third sample on a given sampling day. Then, the amount of each target pyrethroid in the participant's food samples (up to three) was summed over a sampling day (ng/d) and divided by $B$ (body weight $(\mathrm{kg})$ ).

\section{RESULTS}

Table 3 provides the summary statistics for the target pyrethroids and their environmental degradates measured in the duplicatediet solid food samples of $50 \mathrm{Ex}$-R participants over the six-week monitoring period. For the pyrethroids, trans-permethrin (21\%), 
Table 4. Levels of the four most frequently detected pyrethroids in the duplicate-diet solid food samples., ${ }^{\text {a,b }}$

\begin{tabular}{lllllllrr}
\hline Target analyte & $\mathrm{N}^{\mathrm{c}}$ & $\%^{\mathrm{d}}$ & Mean $\pm \mathrm{SD}$ & Min. & 50th & 75th & 90th & 95th $^{\mathrm{f}}$ \\
\hline Bifenthrin & 156 & 100 & $1.8 \pm 2.8$ & 0.06 & 0.63 & 1.7 & 4.7 & 8.2 \\
cis-Deltamethrin & 129 & 100 & $1.9 \pm 2.4$ & 0.12 & 1.0 & 2.4 & 1.1 & 6.2 \\
cis-Permethrin & 145 & 100 & $9.1 \pm 20.0$ & 0.10 & 1.1 & 5.6 & 28.7 & 52.5 \\
trans-Permethrin & 164 & 100 & $8.9 \pm 22.2$ & 0.10 & 0.80 & 4.9 & 25.8 & 4.3 \\
\hline
\end{tabular}

${ }^{a}$ Includes pyrethroids that were detected in $\geqslant 17 \%$ of the food samples (Table 3 ). For each analyte, only values at or above the LOD are presented in this table. ${ }^{b}$ Units $=$ ng/g. ${ }^{c}$ Number of samples. ${ }^{\mathrm{d}}$ Percentage of samples. ${ }^{\mathrm{e}}$ Arithmetric mean and SD. ${ }^{\mathrm{f}}$ Percentile.

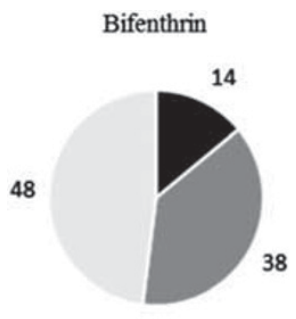

\section{cis-Deltamethrin}

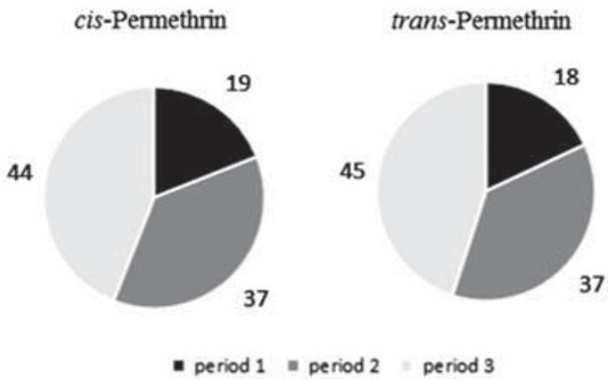

Figure 1. Percentage of duplicate-diet solid food samples with detectable levels of bifenthrin, cis-deltamethrin, cis-permethrin, and trans-

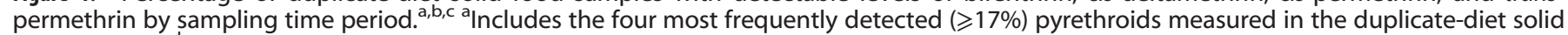
food samples. 'Data are shown for food samples that have detectable levels $(\geqslant L O D)$ of each pyrethroid by time period (period $1=0400-$ 1100 hours, period $2=1100-1700$ hours, and period $3=1700-0400$ hours). ${ }^{c}$ Number of food samples: bifenthrin $(n=156)$, cis-deltamethrin $(n=129)$, cis-permethrin $(n=145)$, and trans-permethrin $(n=164)$.

bifenthrin (20\%), cis-permethrin (19\%), and cis-deltamethrin (17\%) were detected the most often in the food samples. At the 95th percentile, the levels of cis-permethrin $(5.2 \mathrm{ng} / \mathrm{g})$ and transpermethrin $(5.4 \mathrm{ng} / \mathrm{g})$ were at least two times greater compared to the levels of the other pyrethroids $(\leqslant 1.9 \mathrm{ng} / \mathrm{g})$ in the samples. The highest maximum concentration of the pyrethroids occurred for esfenvalerate $(358 \mathrm{ng} / \mathrm{g})$ in one participant's food sample. In comparison to the parent pyrethroids, the degradates were not frequently detected $(<3 \%)$ in the food samples (Table 3 ). MPA (bifenthrin degradate) was not detected in any of these samples. The highest maximum levels of the degradates occurred for transDCCA $(38.3 \mathrm{ng} / \mathrm{g})$ and cis-DCCA $(8.3 \mathrm{ng} / \mathrm{g})$ in the same food sample of one participant.

Table 4 presents the summary statistics for the levels ( $\geqslant L O D$ ) of the four most frequently detected pyrethroids (bifenthrin, cisdeltamethrin, cis-permethrin, and trans-permethrin) in the food samples. Mean levels of cis-permethrin $(9.1 \pm 20.0 \mathrm{ng} / \mathrm{g})$ and transpermethrin $(8.9 \pm 22.2 \mathrm{ng} / \mathrm{g})$ were at least four time greater compared to mean levels of bifenthrin $(1.8 \pm 2.8 \mathrm{ng} / \mathrm{g})$ and cisdeltamethrin $(1.9 \pm 2.4 \mathrm{ng} / \mathrm{g})$ in the food samples. At the 95th percentile, the levels of cis-permethrin $(52.5 \mathrm{ng} / \mathrm{g})$ and transpermethrin $(48.6 \mathrm{ng} / \mathrm{g})$ were at least five times higher than the levels of the other two pyrethroids $(\leqslant 8.2 \mathrm{ng} / \mathrm{g})$. Figure 1 presents the levels of these four pyrethroids in the participant's food samples by sampling time period $(1,2$, or 3$)$. The results show that these four pyrethroids were consistently detected the least often in the food samples during period 1 (4:00 am - 11:00 am) compared with period 2 (11:00 am - 5:00 pm) and period 3 (5:00 pm - $4.00 \mathrm{am})$. These pyrethroids were found the most often in the participants' food samples during period 3, except for cis-deltamethrin (period 2).

The co-occurrence of the target pyrethroids in the food samples of the Ex-R adults over the six-week monitoring period is presented in Figure 2. In this figure, the results show that $49 \%$ of all the food samples contained at least one or more target pyrethroids. In particular, 32\% of the participants' food samples contained one target pyrethroid. In addition, $13 \%$ of the adult food samples contained at least two different target pyrethroids. Only $3 \%$ and $1 \%$ of the food samples had three or four different target pyrethroids, respectively.

Table 5 presents the estimated adults' maximum dietary exposures (ng/day) and maximum dietary intake doses ( $\mathrm{ng} / \mathrm{kg} /$ day) to the target pyrethroid insecticides and compares these estimates to available oral reference doses (RfDs) in the US EPA's Integrated Risk Information System (IRIS). ${ }^{26}$ In IRIS, oral RfDs currently exist only for bifenthrin, $\lambda$-cyhalothrin, cypermethrin, and permethrin. The participants' maximum dietary intake doses were the highest for the combined isomers of cis/trans-permethrin $(2,115 \mathrm{ng} / \mathrm{kg} /$ day $)$, followed by esfenvalerate $(1,784 \mathrm{ng} / \mathrm{kg} / \mathrm{day})$. The estimated adults' maximum dietary intake doses to bifenthrin, $\lambda$-cyhalothrin, cypermethrin, and permethrin were all well below the established oral RfDs in IRIS. 


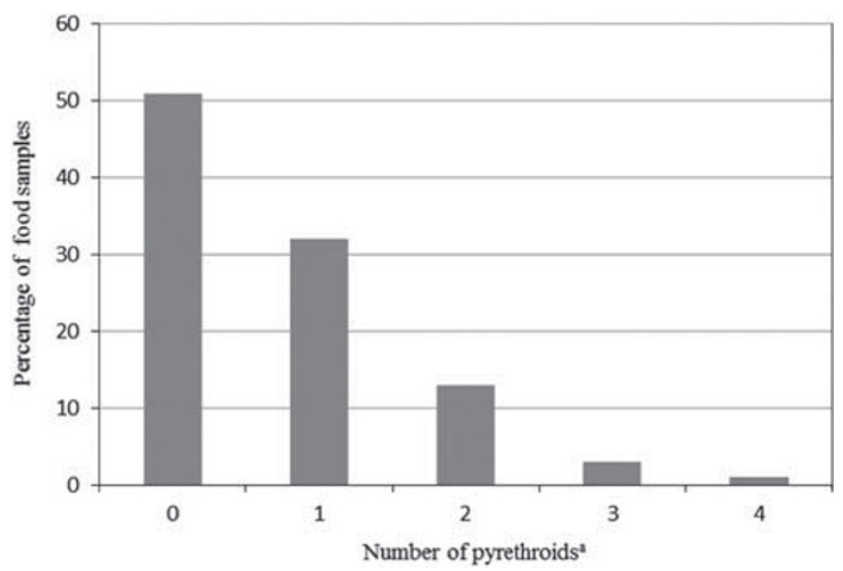

Figure 2. Co-occurrence of the target pyrethroid insecticides in the duplicate-diet solid food samples of Ex-R adults. ${ }^{a}$ cis-and transpermethrin were counted as one pyrethroid insecticide.

Table 5. The estimated adults' maximum dietary exposures and intake doses to the target pyrethroid insecticides. ${ }^{a}$

\begin{tabular}{|c|c|c|c|}
\hline Pyrethroid & $\begin{array}{l}\text { Maximum dietary } \\
\text { exposure (ng/day) }\end{array}$ & $\begin{array}{l}\text { Maximum dietary } \\
\text { dose (ng/kg/day) }\end{array}$ & $\begin{array}{l}\text { Oral } R f D^{\mathrm{b}} \\
\text { (ng/kg/day) }\end{array}$ \\
\hline Bifenthrin & 6206 & 63.1 & 15,000 \\
\hline Cyfluthrin & $31,153^{c}$ & 265 & $-^{d}$ \\
\hline$\lambda$-Cyhalothrin & 7807 & 69.7 & 5000 \\
\hline Cypermethrin & $46,207^{c}$ & 393 & 10,000 \\
\hline cis-Deltamethrin & 205 & 2.93 & - \\
\hline Esfenvalerate & 102,071 & 1784 & - \\
\hline Permethrin $^{\mathrm{e}}$ & 121,000 & 2115 & 50,000 \\
\hline \multicolumn{4}{|c|}{$\begin{array}{l}\text { aEstimates using 24-h duplicate-diet solid food samples (excludes } \\
\text { beverages). }{ }^{b} \text { Oral reference dose (RfD), US EPA's Integrated Risk Information } \\
\text { System (IRIS), http://www.epa.gov/iris/. ' }{ }^{c} \text { Missing food mass data from same } \\
\text { study participant due to laboratory error; used average food mass data of } \\
\text { all study adults by time period. }{ }^{d} \text { Not available in IRIS. }{ }^{\text {e}} \text { Combined cis- and } \\
\text { trans-isomers. }\end{array}$} \\
\hline
\end{tabular}

\section{DISCUSSION}

Previous research has suggested that the diet is likely the major source of exposure to pyrethroids insecticides in the general adult population worldwide. ${ }^{4,5,27}$ Few data exist on the temporal levels of current-use pyrethroid insecticides in the everyday diets of adults. $^{10,12}$ Our study results showed that the individual target pyrethroids were not detected often (1-21\%) in the duplicate-diet solid food samples of 50 Ex-R adults over the 6-week monitoring period. However on a cumulative basis, $49 \%$ of the participants' food samples contained at least one of the target pyrethroids. Based on these data, $100 \%$ of the Ex-R participants were intermittently exposed to several different pyrethroids in their normal diets during the six-week monitoring period. Our study results are similar to Melnyk et al. ${ }^{12,28}$ that also showed low detection frequencies (LOD range $=0.05-0.8 \mathrm{ng} / \mathrm{g}$ ) for bifenthrin $(30 \%)$, cyfluthrin $(4 \%)$, cypermethrin $(11 \%)$, cis-deltamethrin $(1 \%)$, esfenvalerate (10\%), and cis/trans-permethrin (33\%) in 67 solid food samples composited by eating event (breakfast, lunch, dinner, and snack) over several days for nine Hispanic women in Florida USA in 2008. In an earlier study conducted by Riederer et al., ${ }^{10}$ the authors reported higher detection frequencies (LOD range $=0.4-0.8 \mathrm{ng} / \mathrm{g}$ ) than our study for cyfluthrin (59\%), cypermethrin (67\%), cis-deltamethrin (28\%), and cis/trans-permethrin (43\%) in duplicate diet samples (composited by food group) of 12 adults collected over eight sampling days in Georgia USA in 20052006. Interestingly in that study, the authors reported that measureable levels of these pyrethroid residues were found in several different types of foods (i.e., fruits, vegetables, legumes, grains, meats, and dairy). ${ }^{10}$ This information emphasize the importance of collecting total diet samples (i.e., not just produce) from adults to assess their daily dietary exposures to pyrethroids. As data are limited, more research is necessary to determine the cumulative and temporal dietary exposures of adults to currentuse pyrethroid insecticides.

Another important study finding was that the detectable levels of the target pyrethroids substantially varied by sampling time frame (period 1,2, or 3) over a day. The most frequently detected pyrethroids (bifenthrin, cis-deltamethrin, cis-permethrin, and transpermethrin) were found less often in food consumed during period 1 compared to periods 2 and 3 . In support of our study results, Melnyk et al. ${ }^{12,28}$ also reported that the mean intake levels of several different pyrethroids were substantially different by type of adult meal sampled (breakfast, lunch, or dinner). In that study, the authors found that the mean intake levels of permethrin, cypermethrin, and esfenvalerate were the lowest in the participants breakfast meals compared to their lunch/dinner meals. This information suggests that people's eating patterns are likely an important factor influencing their dietary exposures to pyrethroid insecticides over a day.

Few data are available on the estimated dietary intake doses to single or multiple pyrethroid insecticides derived from the actual consumed diets of adults, globally. ${ }^{10}$ Assuming $100 \%$ absorption, our estimated adults' maximum dietary intake doses to bifenthrin $(63.1 \mathrm{ng} / \mathrm{kg} /$ day $), \lambda$-cyhalothrin $(69.7 \mathrm{ng} / \mathrm{kg} /$ day $)$, cypermethrin (393 ng/kg/day), and permethrin (2,115 ng/ $\mathrm{kg} /$ day) were all well below the corresponding oral RfDs listed in IRIS (Table 5). ${ }^{26}$ Since oral RfDs are not currently available for cyfluthrin, cis-deltamethrin, or esfenvalerate in IRIS, ${ }^{26}$ we could not determine if the estimated adults' dietary intake doses to these insecticides (Table 5) were below a level of concern. Only one other smaller duplicate-diet study was found by Reiderer et al. ${ }^{10}$ that reported estimated dietary intake doses to cypermethrin $(1400 \mathrm{ng} / \mathrm{kg} /$ day $)$, cisdeltamethrin ( $2700 \mathrm{ng} / \mathrm{kg} /$ day), and permethrin $(1500 \mathrm{ng} / \mathrm{kg} /$ day $)$ for 12 adults in Georgia USA in 2005-2006.

Few published studies have measured the concurrent levels of pyrethroids and their environmental degradates (commonly used as urinary biomarkers) in individual food items (i.e., produce) $)^{7,11}$ and none in duplicate-diet samples. Our study results showed that 3PBA, 4-F-3-PBA, cis-DBCA, cis-DCCA, trans-DCCA, and MPA were not frequently detected $(<3 \%)$ in the duplicate-diet solid food samples of 50 Ex-R participants over the six-week monitoring period. This information suggests that these pyrethroid degradates were probably not present in sufficient levels in the diet to substantially influence the Ex-R adults' urinary pyrethroid biomarker concentrations. Our results are in agreement with $\mathrm{Li}$ et al. ${ }^{7}$ that recently reported measureable levels of several different pyrethroids in samples of apples, grapes, and lettuce purchase from local grocery stores in Durham NC, but only the pyrethroid degradates, cis-DCCA and trans-DCCA, occurring in one lettuce sample.

Our study had some limitations. Because of participant burden and budget constraints, the Ex-R study only collected duplicatediet solid food samples (which excluded beverages). Therefore, it is possible that we may have underestimated the total daily dietary exposures and intake doses of the Ex-R adults to these individual pyrethroids. However, previous research has indicated that beverages were a minor contributor to the dietary exposure of adults to pyrethroid insecticides. ${ }^{6,9,29}$ Another study limitation was that the Ex-R participants' solid food samples were collected over three, consecutive time frames each sampling day, so we were unable to determine the specific food items that had detectable levels of the pyrethroid residues. 


\section{CONCLUSIONS}

On basis of the food sample data, our study results showed that the Ex-R adults were likely intermittently exposed to several different pyrethroid insecticides in their diets during the six-week monitoring period in NC in 2009-2011. In addition, the results suggests that the measured pyrethroid degradates (3-PBA, 4-F-3PBA, cis-DBCA, cis-DCCA, and trans-DCCA) were likely not present in sufficient levels in the diet to substantially impact the adults' urinary biomarker concentrations. ${ }^{30}$

\section{CONFLICT OF INTEREST}

The authors declare no conflict of interest.

\section{ACKNOWLEDGEMENTS}

We thank Carry Croghan, Paul Jones, Fu-Lin Chen, Lillian Alston, Richard Walker, Erik Andersen, Jim Starr, Scott Clifton, and Scott Rhoney for their technical assistance in this study. We would also like to thank Joseph Evans (Alion) for homogenizing the food samples.

\section{DISCLAIMER}

This United States Environmental Protection Agency through its Office of Research and Development has provided administrative review of this article and approved it for publication.

\section{REFERENCES}

1 Elbert A, Haas M, Springer B, Thielert W, Nauen R. Applied aspects of neonicotinoid uses in crop protection. Pest Manag Sci 2008; 64: 1099-1105.

2 Saillenfait AM, Ndiaye D, Sabate JP. Pyrethroids: exposure and health effects an update. Int J Hyg Envir Heal 2015; 218: 281-292.

3 USEPA (United States Environmental Protection Agency). Pyrethrins/ Pyrethroids Cumulative Risk Assessment. Available at: http://epa.gov/pesticides/cumulative/ common mechgroups/htm\#pyrethrins, 2011. Accessed on 29 July 2015.

4 Riederer AM, Bartell SM, Barr DB, Ryan PB. Diet and nondiet predictors of urinary 3-phenoxybenzoic acid in NHANES 1999-2002. Environ Health Perspect 2008; 116: 1015-1022.

5 Barr DB, Olsson AO, Wong LY, Udunka SO, Baker SE, Whitehead RD et al. Urinary concentrations of metabolites of pyrethroid insecticides in the general US population: National Health and Nutrition Examination Survey 1999-2002. Environ Health Perspect 2010; 118: 742-748.

6 Morgan MK, Sheldon LS, Croghan CW, Jones PA, Chuang JC, Wilson NK. An observational study of 127 preschool children at their homes and daycare centers in Ohio: Environmental pathways to cis- and trans-permethrin exposure. Environ Res 2007; 104: 266-274.

7 Li W, Morgan MK, Graham S, Starr JM. Measurement of Pyrethroids and Their Environmental Degradates in Fresh Fruits and Vegetables using a Modification of the Quick Easy Cheap Effective Rugged Safe (QuEChERS) Method. Talanta 2016; 151: 42-50.

8 Chuang JC, Wilson NK. Multiresidue analysis of organophosphate and pyrethroid pesticides in duplicate-diet solid food by pressurized liquid extraction. $J$ Environ Sci Health B 2011; 46: 41-40.

9 Lu C, Schenck FJ, Pearson MA, Wong JW. Assessing children's dietary pesticide exposure: Direct measurement of pesticide residues in 24-hr duplicate food samples. Environ Health Perspect 2010; 118: 1625-1630.

10 Riederer AM, Hunter RE, Hayden SW, Ryan PB. Pyrethroid and organophosphorus pesticides in composite diet samples from Atlanta, USA adults. Environ Sci Technol 2010; 44: 483-490.

11 Chen L, Zhao T, Pan C, Ross JH, Krieger RL. Preformed biomarkers including dialkylphosphate (DAPs) in produce may confound biomonitoring in pesticide exposure and risk assessment. J Agric Food Chem 2012; 60: 9342-9351.

12 Melnyk L, McCombs M, Brown GG, Raymer JJ, Nishioka MG, Buehler S et al. Community duplicate diet methodology: A new tool for estimating dietary exposures to pesticides. J Environ Monit 2012; 14: 85-93.
13 USDA (United States Department of Agriculture). Pesticide Data Program. Available at: http://www.ams.usda.gov/datasets/pdp. 2014. Accessed on 13 January 2016.

14 USFDA (United States Food and Drug Administration). Total Diet Study. 2004-2005. Available at: http://www.fda.gov/Food/FoodScienceResearch/TotalDietStudy/ default.htm. Accessed on 13 January 2016.

15 Leng $\mathrm{G}$, Kuhn $\mathrm{KH}$, Idel $\mathrm{H}$. Biological monitoring of pyrethroids in blood and pyrethroid metabolites in urine; Applications and limitations. Sci Total Environ 1997; 199: 173-181.

16 Ratelle M, Cote J, Bouchard M. Toxicokinetics of permethrin biomarkers of exposure in orally exposed volunteers. Toxicol Lett 2015; 232: 369-375.

17 Eadsforth CV, Bragt PC, Van Sittert NJ. Human dose-excretion studies with pyrethroid insecticides cypermethrin and alphacypermethrin: Relevance for biological monitoring. Xenobiotica 1988; 18: 603-613.

18 CDC (Centers for Disease Control and Prevention). Fourth national report on human exposure to environmental chemicals. 2009. Available at: http://www.cdc. gov/ exposurereport/. Accessed on 11 August 2015.

19 McKelvey W, Jacobson JB, Kass D, Barr DB, Davis M, Calafat AM et al. Populationbased biomonitoring of exposure to organophosphate and pyrethroid pesticides in New York City. Environ Health Perspect 2013; 121: 1349-1356.

20 Trunnelle KJ, Bennett DH, Tulve NS, Clifton MS, Davis MD, Calafat AM et al. Urinary Pyrethroid and Chlorpyrifos Metabolite Concentrations in Northern California Families and Their Relationship to Indoor Residential Insecticide Levels, Part of the Study of Use of Products and Exposure Related Behavior (SUPERB). Environ Sci Technol 2014; 48: 1931-1939.

21 Morgan MK, Sobus JR, Boyd-Barr D, Croghan CW, Chen F, Walker R et al. Temporal variability of pyrethroid metabolite levels in bedtime, morning, and 24-hr urine samples for 50 adults in North Carolina. Environ Res 2016; 144: 81-91.

22 Anastassiades M, Lehotay SJ, Stajnbaher D, Schenck FJ. Fast and easy multiresidue method employing acetonitrile extraction partitioning and dispersive solid phase extraction for determination of pesticide residues in produce. J AOAC Int 2003; 86 412-431.

23 Lehotay SJ, Mastovska K, Yun SJ. Evaluation of two fast and easy methods for pesticide residue analysis in fatty food matrixes. J AOAC Int 2005; 88: 630-638.

24 Rosenblum L, Hieber T, Morgan J. Determination of pesticides in composite dietary samples by gas chromatography/mass spectrometry in the selected ion monitoring mode by using a temperature-programmable large volume injector with preseparation column. J AOAC Int 2001; 84: 891-900.

25 Verbovsek T. A comparison of parameters below the limit of detection in geochemical analyses by substitution methods. Materials Geoenviron 2011; 58: 393-404.

26 IRIS (Integrated Risk Information System). 2015. Available at: http://www.epa.gov/ iris/. Accessed on 21 August 2015.

27 Schettgen T, Heudorf U, Drexler H, Angerer J. Pyrethroid exposure of the general population - is this due to diet? Toxicol Lett 2002; 134: 141-145.

28 Melnyk $\amalg$, Xue J, Brown GG, McCombs M, Nishioka M, Michael LC. Dietary intakes of pesticides based on community duplicate diet samples. Sci Total Environ 2014; 468-469: 785-790.

29 Morgan MK, Sheldon LS, Croghan CW, Chuang JC, Lordo R, Wilson NK et al. A Pilot Study of Children's Total Exposure to Persistent Pesticides and Other Persistent Organic Pollutants (CTEPP). United States Environmental Protection Agency: Washington, DC, USA, 2004 EPA/600/R-041/193.

30 Matuszewski BK, Constanzer ML, Chavez-Eng CM. Strategies for the assessment of matrix effect in quantitative bioanalytical methods based on HPLC-MS/MS. Anal Chem 2003; 75: 3019-3030.

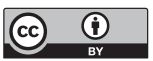

This work is licensed under a Creative Commons Attribution 4.0 International License. The images or other third party material in this article are included in the article's Creative Commons license, unless indicated otherwise in the credit line; if the material is not included under the Creative Commons license, users will need to obtain permission from the license holder to reproduce the material. To view a copy of this license, visit http://creativecommons.org/licenses/ by/4.0/

(c) The Author(s) 2018

Supplementary Information accompanies the paper on the Journal of Exposure Science and Environmental Epidemiology website (http:// www.nature.com/jes) 